\title{
Archiving Qualitative and Qualitative Longitudinal Social Sciences Data In the Czech Republic
}

by Tomáš Č́žek'

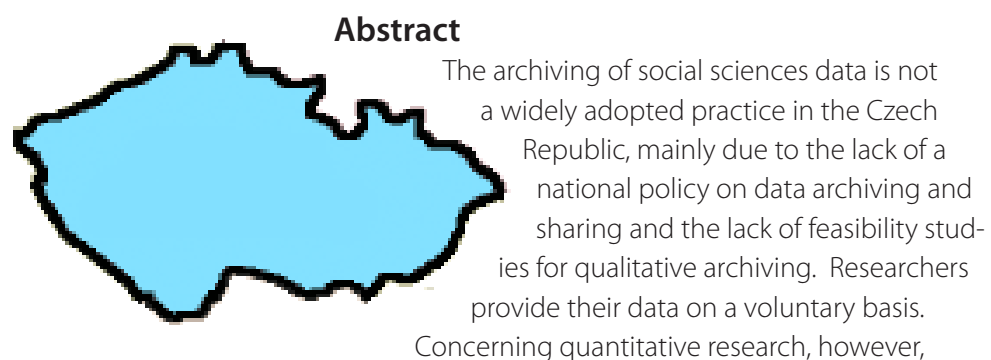

hundreds of projects have been carried out that represent a large share of research projects in the Czech Republic. The quantitative section of our archive is well-developed and serves hundreds of users, mainly students. Yet, the situation in the field of qualitative research is completely different. Archiving

is not a component part of the research culture and this will hardly change in the near future. The practice of the use of informed consent (particularly in a standardized form allowing archiving) is far from being adopted. Therefore, our archive's qualitative data library has only a limited number of data files and, as a consequence, of users.

Keywords: Archive, Czech, Qualitative data, Research infrastructure
Existing qualitative archiving infrastructure The infrastructure consists solely of our archive, 'Medard', which, as an independent data library, is a part of The Sociological Data Archive, Institute of Sociology at the Academy of Sciences of the Czech Republic.

At present, the archive provides a small number of data files (seven), consisting of several dozens of completed interviews. Several other data files are currently in the process of being archived and will be made available in the near future. The data archive is used about ten times a year mainly by the students of social sciences and, rarely, also by researchers. The archive functions as an electronic library, and this is why the data are archived in a digital

\section{Qualitative research has been developing only for twenty years in the Czech Republic}

form. Presently, there are transcriptions of interviews and also audio and video recordings deposited there.

As a part of the Institute of Sociology, the archive is financed through its research arm. However, there is only a small budget covering half of one employee's salary. The archive also provides extended electronic counseling in 
Czech related to qualitative research, as well as basic information in English, on its web pages (See http://medard.soc.cas.cz).

Qualitative research has been developing only for twenty years in the Czech Republic. To the best of the author's knowledge, longitudinal research projects are not widely performed. In the Czech Republic, the only instances of such research were longitudinal documentary films that are available in the form of compact works of art with video recordings (dozens and even hundreds of hours of recorded life histories) but these are not available for studying purposes.

However there is a tradition of oral historical work, notably as a study of life histories somehow related to significant events in Czech history. The Oral History Center has already made several dozens of interviews available as transcriptions in the form of books as well as in an authentic audio format obtainable from the Center (see http://www.coh.usd. (as.cz/).

\section{Development planning}

The archive has existed already for many years. So far, however, it has been a rarely used infrastructure. It is necessary to extend and revise the practice of informed consent that would allow archiving. The author has been giving lectures at universities and conferences aimed at promoting and supporting the practices, yet the result is only limited. In the near future, we are planning to establish a method of data handling and archiving in cooperation with Charles University in Prague that will be binding for the students of sociology conducting qualitative research. The archive is also involved in the project "Czech Sociology 1945-1968 Oral History". Interviews performed within it are archived in the form of interview transcriptions, audio and video recordings.

Considering the necessity of significantly extending the contents of the data archive, it will be necessary to perform an extensive study of qualitative research in the Czech Republic and, based on its findings, to identify the files suitable for archiving.

The cooperation with CESSDA or IASSIST should, first of all, provide us with the opportunity to become acquainted with "good practice" at other workplaces and, in this sense, inspire our further work. This would mean, for example, support for study visits to established workplaces, or perhaps collaborative involvement in international projects.

\section{Notes}

1. Tomáš Čížek

tomas.cizek@soc.cas.cz

Institute of Sociology, Academy of Sciences of the Czech Republic

The Sociological Data Archive (SDA)

Jilská 1, 11000 Praha 1Prague, Czech Republic

http://Medard.soc.cas.cz 